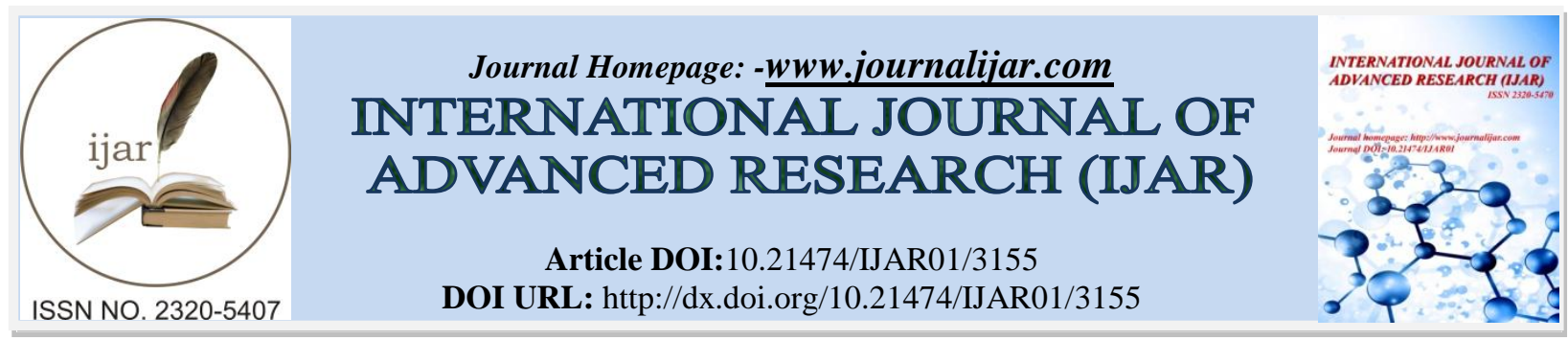

RESEARCH ARTICLE

\title{
A SURVEY ON TASK SCHEDULING MODEL IN CLOUD COMPUTING USING OPTIMIZATION TECHNIQUE.
}

\author{
A. Nandhini ${ }^{1}$, S.Radha ${ }^{1}$, T.V.Pavithra ${ }^{1}$ and Dr G.Umarani Srikanth ${ }^{2}$. \\ 1. Students, Department of computer science, S.A.Engineering College, Anna University \\ 2. Professor \& HOD, Department of computer science, S.A.Engineering College, Anna University
}

\section{Manuscript Info}

Manuscript History

Received: 13 December 2016

Final Accepted: 19 January 2017

Published: February 2017

Key words:-

Ant Colony Optimization, makespan, performance.

\begin{abstract}
Task scheduling is the most important part of cloud computing. To optimize the system, the tasks have to be scheduled in an efficient manner. A scheduling algorithm must be efficient in a way that it improves the performance of the system. The primary goal of task scheduling algorithm is to reduce the makespan and to increase resource utilization. In this paper a task scheduling model using various algorithms has been analyzed. These algorithms take into consideration of various parameters and improve the system.
\end{abstract}

Copy Right, IJAR, 2017,. All rights reserved.

\section{Introduction:-}

Cloud Computing:-

Cloud computing is a shared pool of the various configurable computing resources (e.g. server, storage, network, services, and applications) that can be accessed remotely without owning the entire cloud. Cloud scheduling is the scheduling of incoming tasks to the available $\boldsymbol{n}$ resources. There are various scheduling algorithms and the traditional scheduling algorithms only aims at reducing the total execution time of the system. The meta-heuristic algorithms like Ant Colony Optimization (ACO) and Particle Swarm Optimization (PSO) tend to be efficient than traditional scheduling algorithms.

\section{Need for Cloud Scheduling:-}

Many users access the cloud at the same time and it is not possible to allocate each task manually. Due to increase in demand for cloud resources, the resources need to be allocated to each task in an efficient manner. So there is a need for cloud scheduling to schedule the tasks to resources efficiently.

Max-Min algorithm select tasks with maximum execution time on a faster available machine that is capable of giving minimum completion time. The disadvantage of this algorithm is that the tasks with minimum execution time are delayed. Hence this algorithm is not efficient for scheduling. Genetic algorithm is used to find the optimal sequence of tasks. By executing tasks in that sequence the makespan of the tasks gets reduced. PSO algorithm follows the behavior of bird searching for foods. It searches the perfect place to schedule the data by using the random number which is used to search the location. Ant colony algorithm follows the behavior of ants. It uses the pheromone laid on the path to travel. It will be disappeared after some time so that new paths are formed automatically. Bee colony algorithm follows the behavior of bees called foraging. It is used to find the feasible path for scheduling problem. The bees are divided into different groups and do the job like that in the bee colony.

Corresponding Author:-A.Nandhini.

Address:-Students, Department of computer science, S.A.Engineering College, Anna 


\section{Related Works:-}

Liji Jacob [1] proposed the BAT algorithm for scheduling in the tasks in the cloud. This algorithm is inspired by the behavior of bats. Bats use echoes to sense food/prey and barriers in the darkness. They fly randomly with fixed frequency and loudness. Initially, bat population and its echolocation parameters are initialized. Candidate micro bats are then generated and it is evaluated. After evaluation, the echolocation parameters are updated. It is repeated until there is a feasible design. This algorithm is compared with other optimization algorithms and it has high accuracy, high efficiency, and high convergence rate.

UmaraniSrikanth et al. [2] proposed that in cloud computing it is very important to schedule the task correctly. If the task scheduling algorithm is used to schedule means it will take more time for allocation. In order to avoid that here, they are trying to predict a schedule for the new task set without running the task scheduling algorithm. For that, they are using the ACO, a swarm intelligence model to reduce the scheduling time. Here they use the utilization matrix.

SumandeepAujla et al. [3] proposed the hybrid Cuckoo algorithm. This algorithm is a combination of Genetic Algorithm (GA) and Cuckoo algorithm. First, the tasks are scheduled by genetic algorithm and the output from GA is given as input to the Cuckoo algorithm. The results are proven to be better than GA and Cuckoo algorithm in terms of energy, execution time and resource utilization.

Awad et al. [4] proposed the Load Balancing Mutation Particle Swarm Optimization (LBMPSO) algorithm which is an enhanced form of PSO algorithm. This model is proposed for allocation of tasks to virtual machines with maintaining the reliability. The tasks that are failed to schedule are rescheduled using LBMPSO, thus achieving reliability. This algorithm is compared with standard PSO and few other algorithms. It is found that this algorithm reduces the execution time, cost, transmission time and helps in achieving the load balancing between tasks and virtual machines. It provides a reliable and good distribution of resources when compared with other algorithms.

NimaJafariNavimipour [5] proposed the artificial bee colony algorithm. This algorithm works based on the foraging behavior of honey bees. This algorithm consists of employed, onlooker and scout bees. Each employed bee searches for the new food source in the neighborhood of its current food source. The onlooker bee evaluates the nectar information from all employed bee and chooses the best. The scout bees determine the abandoned sources and replace it with randomly produces new food sources. Food source represents the possible solution and the nectar amount represents the quality of the solution. This algorithm is proved to be better in terms of-of task execution time and waiting time.

Ramya et al.[6] proposed a cost efficient algorithm for task scheduling. It uses two scheduling heuristic. The first heuristic uses the concept of Pareto dominance to generate a cost efficient task schedule based on the execution time of the tasks and the monetary charges of VM's. The second heuristic complements to the first heuristic and attempts to minimize the monetary costs of non-critical tasks.

Durga Lakshmi et al. [7] proposed the genetic algorithm which is a heuristic search technique that produces an optimal solution of tasks. This algorithm mainly aims to find the best sequence of tasks to execute to optimize the overall waiting time of the system. The request from the Client User (CU) is stored in the Request Queue (RQ). Then GA Module Queue Sequencer (GAQS) interchanges the position of the tasks so that the waiting time of the tasks is less. The sequence of tasks is stored in buffer queue and then it is executed by the Job Scheduler (JS). This algorithm is compared with tradition scheduling algorithm and it increases the throughput of the system and can be implemented to increase the performance of the system.

KokKonjaang et al. [8] proposed the Max-Min algorithm to efficiently optimize the task scheduling by allocating the tasks with maximum execution time on a faster available machine. This algorithm aims at the first allocation of tasks with maximum execution time before assigning the tasks with minimum execution time for the purpose of minimizing make span. This enhanced algorithm is compared with Max-Min and Data Aware algorithms. It is found that it provides efficient resource allocation and reduces the make span. The drawback of this algorithm is that there will be a delay in execution of tasks with minimum execution time.

Leila Ismail et al. [9] proposed the Energy-Aware Task Scheduling (EATS) model which aims to increase the efficiency of the application and to reduce the energy consumption. In this model, the big data is divided and 
scheduled in the cloud for distributed processing by taking into consideration of both the performance and energy consumption. They have taken the application to be a divisible load application so that it is divided into independent tasks and assigned to distributed resources. The distributed processing consumes less energy compared to sequential execution. It is shown that the ratio of consumption of energy in a fully utilized server to its consumption of energy when it is idle is 1.3 which says that the server should be fully utilized to reduce energy consumption and increase performance.

Hussin M. Alkhashai et al. [10] proposed an algorithm which is a combination of Particle Swarm Optimization (PSO), the Best-Fit (BF), and Tabu-Search (TS) algorithms called BFPSOTS. The BF algorithm is used to generate an initial population of standard PSO, the Tabu-Search (TS) algorithm has been used with PSO to avoid the trap of local optimality which occurred when using the standard PSO. The cloudsim tool is used for the implementation of this hybrid algorithm. This algorithm reduces the makespan, cost and increases the resource utilization.Butstill, this improvement is achieved at the expense of fulfillment of the time complexity.

ShengjunXue et al. [11] proposed the experiment and analysis of task scheduling of workflow under the cloud computing by using the particle swarm optimization. It is used to achieve the minimum cost of users. Workflow represents the logic and rules that how to organize the front and rear works together in the work process. Here they use the service cost optimization algorithm based on PSO (PSO-SC). This algorithm makes use of the matrix to solve the problem with the help of graph with in-degree and out-degree. In this, it not only decreases the total time it also adjusts the execution task with the help of virtual machines in the data centers.

Parminder Singh et al. [12] proposed an improved and adaptive firefly algorithm for efficient task scheduling in the cloud. This algorithm has two aspects. First is the development of the cloud framework for job scheduling while the second is the development of firefly algorithm which can be applied to the cloud to improve the task scheduling. This algorithm is compared with other meta-heuristic algorithms and it provides better results than others.

\section{Conclusion:-}

By using ACO algorithm, a swarm intelligence model the scheduling time is reduced, pheromone value used will be disappeared automatically and the new path is formed for scheduling the task. Hence, it will increase the performance and efficiency of the model. This model will automatically schedule the tasks without using the task scheduling algorithm so that scheduling time is reduced.

\section{References:-}

1. Liji Jacob, "Bat Algorithm for Resource Scheduling In Cloud Computing", International Journal For Research In Applied Science And Engineering Technology (IJRASET), Vol. 2 Issue IV, pp.53-57 April 2014.

2. G. UmaraniSrikanth, V. Uma Maheswari, A. P. Shanthi, Arul Siromoney, "Task Scheduling Model", Indian Journal of Science and Technology, Vol 8(S7),pp. 33-42, April 2015.

3. SumandeepAujla, AmandeepUmmat, "Task scheduling in Cloud Using Hybrid Cuckoo Algorithm", International Journal of Computer Networks and Applications (IJCNA) Volume 2, Issue 3, pp.144-150 May June (2015).

4. A.I.Awad, N.A.El-Hefnawy, H.M.Abdel_kader, "Enhanced Particle Swarm Optimization for Task Scheduling In Cloud Computing Environments ", International Conference on Communication, Management and Information Technology (ICCMIT 2015) pp.920-929.

5. NimaJafariNavimipour, "Task scheduling in the Cloud Environments based on an Artificial Bee Colony Algorithm", Proceedings of 2015 International Conference on Image Processing, Production and Computer Science (ICIPCS'2015) Istanbul (Turkey), June 3-4, 2015 pp. 38-44.

6. G.Ramya, P.Keerthika, P. Suresh, and M.Sivaranjani, "Optimized Scheduling Of Tasks Using Heuristic Approach With Cost-Efficiency In Cloud Data Centers", International Journal of Scientific \& Engineering Research, Volume 7, Issue 2,pp.208-213 February-2016.

7. R. Durga Lakshmi, N. Srinivasu, "A dynamic approach to task scheduling in cloud computing using genetic algorithm", Journal of Theoretical and Applied Information Technology 20th March 2016.pp.124-135 Vol.85. No.2. 
8. J. KokKonjaang, J.Y. Maipan-uku, Kumangkem Kennedy Kubuga, "An Efficient Max-Min Resource Allocator and Task Scheduling Algorithm in Cloud Computing Environment ", International Journal of Computer Applications (0975 - 8887) Volume 142 - No.8, pp.25-30 May 2016.

9. Leila Ismail, Abbas Fardoun, "EATS: Energy-Aware Tasks Scheduling in Cloud Computing Systems", The 6th International Conference on Sustainable Energy Information Technology pp.870-877(SEIT 2016).

10. Hussin M. Alkhashai, Fatma A. Omara, "An Enhanced Task Scheduling Algorithm on Cloud Computing Environment", International Journal of Grid and Distributed Computing Vol. 9, No. 7, pp.91-100 (2016).

11. ShengjunXue, Wenling Shi, and XiaolongXu, "A Heuristic Scheduling Algorithm based on PSO in the Cloud Computing Environment", International Journal of u- and e- Service, Science, and Technology, Vol.9, No. 1 pp.349-361(2016).

12. Parminder Singh, AmandeepKaur, "Efficient Task Scheduling Over Cloud Computing with An Improved Firefly Algorithm", International Journal of Engineering Development and Research, Volume 4, Issue 2, pp.1514-1518(2016). 